\title{
articles
}

\section{Hopelessness, depression and suicidal ideation in HIV-positive persons}

\author{
R D Govender, BSc, MB ChB, MFamMed \\ Department of Family Medicine, University of Kwa-Zulu Natal
}

L Schlebusch, MA (Clin Psych), MMedSc (Psych) (Cum Laude), PhD, C Psychol (UK), FRSPH (UK)

Department of Behavioural Medicine, University of KwaZulu-Natal

Background and objectives. HIV/AIDS and suicidal behaviour are major public health concerns. The aim of this study was to examine the relationship between hopelessness, depression and suicidal ideation in HIV-infected persons.

Methods. The sample consisted of all adult volunteers attending a voluntary counselling and testing (VCT) HIV clinic at a universityaffiliated state hospital. Suicidal ideation and depression were measured using the Beck Hopelessness Scale (BHS) and the Beck Depression Inventory (BDI), respectively, at two intervals, viz. 72 hours and 6 weeks after HIV diagnosis.

Results. Of the 156 patients who tested positive for HIV, 32 (20.5\%) had a hopelessness score of 9 or above on the BHS and 130 patients (82.8\%) were depressed according to the BDI at 72 hours after diagnosis. Of the 109 patients assessed 6 weeks after diagnosis, 32 (28.8\%) had a hopelessness score of $>9$ on the $\mathrm{BHS}$ and 86 (78.2\%) were depressed according to the BDI. A moderately positive correlation at both time periods was found between hopelessness and depression. A ROC analysis showed optimal sensitivity, indicating that the HIV-positive depressed patients were at risk for suicidal behaviour.

Conclusion. The significant correlations between hopelessness, depression and suicidal ideation are important markers that should alert healthcare professionals to underlying suicide risks in HIV-positive patients. Early recognition of this and suicide prevention strategies should be incorporated into the treatment offered at VCT HIV clinics.

While suicidal ideation has been extensively studied in the general and medical population, ${ }^{1,2}$ its relationship with HIV infection is less well documented. This is of concern, since the psychological responses to an HIV-positive result can be wide ranging and include feelings of hopelessness, helplessness, anxiety, worthlessness and depression and suicidal behaviour.-5 Recognising this in a biopsychosocial context is paramount to understanding the relationship between the HIV/AIDS pandemic and the increased prevalence of suicidal behaviour in South Africa., ${ }^{4,5}$ The association between hopelessness, depression and suicide has been well documented. ${ }^{1,5}$ Hopelessness, being a feeling of despair and extreme pessimism about the future, forms part of Beck's 'cognitive triad' $^{\prime 6}$ and cognitive theory of depression. ' Central to depression are negative thoughts generated by dysfunctional beliefs, ${ }^{7}$ which in part may trigger suicidal behaviour in HIV-positive patients. ${ }^{5}$

Depression has been identified as a high-risk factor for suicidal behaviour, and a significant percentage of all depressed persons may die by suicide. The lifetime risk for suicide in the general South African population has been found to be about 17\%.5-10 Likewise, studies in Africa have reported high rates of depressive symptoms and suicidal behaviour in HIV-infected persons, $5,11,12$ with the lifetime prevalence for depression ranging from $22 \%$ to $45 \%$ in this population. ${ }^{13}$ The prevalence of depression has been reported to be almost twice as high in HIV-positive than in HIV-negative persons. ${ }^{13}$ Nevertheless, HIV-related suicidal behaviour is a complex phenomenon $n^{4-5,14-15}$ that requires further research. It is against this backdrop that the present study aimed to explore the relationship between hopelessness, depression and suicidal ideation in a patient cohort recently diagnosed as HIV positive.

\section{Methods \\ Sample}

The sample comprised 156 HIV-positive patients assessed 72 hours after diagnosis, and 109 seropositive patients assessed at 6 weeks' follow-up. This sample was obtained from patients attending the voluntary counselling and testing (VCT) HIV clinic at a universityaffiliated state hospital in Durban, South Africa. The study was conducted over a 3-month period. The recruited patients voluntarily signed informed consent and were advised not to answer questions they considered intrusive or sensitive. Patients who tested HIV negative were excluded from the analyses for purposes of this study. The study was approved by the Biomedical Research Ethics Committee of the University of KwaZulu-Natal, and permission to conduct it was granted by the relevant health institution.

\section{Instruments}

The Beck Hopelessness Scale (BHS) and the Beck Depression Inventory (BDI) were used in the study. They were considered appropriate given that they were developed by Beck within the context of his cognitive theory of depression, and their excellent internal consistency, reliability and concurrent validity have been well established across a wide variety of samples. The $\mathrm{BHS}$ is designed to measure negative expectations and feelings of hopelessness about the future and has been used in both inpatients ${ }^{1}$ and outpatients. ${ }^{16}$ Feelings of hopelessness play a pivotal role in the development of negative cognitions, leading to the belief that suicide is the only option to escape unbearable psychological pain. ${ }^{5,17-18}$ According to the results from empirical studies, elevated scores on the BHS are significantly related to suicidal ideation, which can be used to predict eventual suicidal behaviour. ${ }^{1,16-17}$ The scale consists of items that are endorsed as either true or false, with 9 of 
the items keyed false and 11 keyed as true. The answers are totalled to obtain a hopelessness score ranging from 0 to $20 .{ }^{16} \mathrm{As}$ in other research, ${ }^{1,16}$ in this study we used a cut-off score of 9 and above on the BHS to identify suicidal ideation in the HIV-infected patients studied. The BDI consists of 21 items, each with 4 statements that reflect gradations in the intensity of a particular depressive symptom. ${ }^{1720}$ The instrument is composed of items relating to symptoms of depression, including feelings of hopelessness. The first two-thirds of the items rate emotional symptoms and the remaining third physical symptoms. We used the standard cutoff scores empirically derived by the test developer, which are as follows: 0 - 9 indicates minimal depression, 10 - 18 mild depression, 19 - 29 moderate depression, and 30 - 63 severe depression. ${ }^{20}$

Previous studies ${ }^{19,21-23}$ have paid close attention to item 7 on the BHS and item 9 on the BDI in examining suicide risk, and the reliability and validity of using these items to assess suicidal ideation. On item 7 of the BHS, patients have to endorse 'My future looks dark', and on item 9 on the BDI choose between 'I don't have any thoughts of killing myself', 'I have thoughts of killing myself, but I would not carry it out','I would like to kill myself' and 'I would like to kill myself if I had the chance'. Therefore, in line with other research, we decided to focus on these two items in the present study in order to assess suicidal ideation.

\section{Statistical analyses}

SPSS version 15.0 (SPSS Inc., Chicago, III., USA) was used to analyse the data. A $p$-value $<0.05$ was considered statistically significant. Pearson's correlation coefficient was used to assess the presence and strength of the relationship between BDI scores and suicidal ideation at 72 hours and 6 weeks after HIV diagnosis. The chi-square or Fisher's exact probability tests, as appropriate, were used to determine associations between responses to individual items on the $\mathrm{BHS}$ and $\mathrm{BDI}$ and suicidal ideation.

Receiver operating characteristic (ROC) analyses were used to determine the sensitivity and specificity optimal of cut-off points on the $\mathrm{BDI}$ to predict suicidal ideation. ROC analysis is based on the signal detection theory and involves plotting the sensitivity against the false-positive fraction for every cut-off point on a measure. It is used to evaluate the discriminative performance of a screening test in distinguishing cases from non-cases, and yields a summary measure, namely the area under the curve (AUC). The AUC indicates the screening instrument's ability to discriminate between cases and non-cases, with a perfectly accurate discrimination resulting in an AUC of 1.00. The area under the ROC curve (AUC) could be regarded as the probability of correct prediction. ${ }^{2425} \mathrm{ROC}$ analysis was used to determine the sensitivity and specificity of cut-off points on the BDI to predict suicidal ideation. In establishing cut-off points on the BDI which would optimise sensitivity and specificity, it was decided that ideally the test should be more sensitive than specific to identify as many probable suicidal patients as possible. In this regard, according to Beck et al. ${ }^{16}$ sensitivity is paramount to suicide prediction, on which our rationale for maximising sensitivity was based in the present study.

\section{Results}

Thirty-two patients (20.5\%) had a hopelessness score of 9 or more on the BHS 72 hours after HIV diagnosis and 32 (28.8\%) had a score in this range at 6 weeks. One hundred and thirty patients (82.8\%) were depressed according to the BDI scores 72 hours after diagnosis, and 86 (78.2\%) were depressed at 6 weeks. Pearson's correlation coefficients for hopelessness and depression scores were $0.556(p<0.001)$ at 72 hours and $0.625(p<0.001) 6$ weeks after diagnosis, illustrating a moderately positive correlation at both time periods.

The mean scores for item 7 on the BHS were 0.561 at 72 hours and 0.421 at 6 weeks. The mean scores for item 9 on the BDI were 0.154 at 72 hours and 0.0766 weeks later. Table 1 depicts a significant association between the responses to item 9 on the BDI and suicidal ideation with $p=0.036$ at 72 hours and $p=0.008$ at 6 weeks, in that $36 \%$ and $66 \%$ of the patients have evidence of suicidal ideation on both the BDI and BHS as defined by the standard cut-off scores at 72 hours and 6 weeks respectively.

Table 2 shows a statistically significant association $(p=0.002)$ in that at 72 hours, $28 \%$ of the respondents who endorsed item 7 on the BHS had a score sufficiently high to predict suicidal ideation, compared with only $7 \%$ who did not endorse that item. Table 2 also shows that at 6 weeks there was a statistically significant association $(p<0.001)$ between item 7 on the BHS and a predisposition for suicidal ideation (i.e. $57 \%$ of the respondents compared with none endorsed this item).

Table 1. The association between suicidal ideation on the BHS scores and item 9 on the BDI 72 hours and 6 weeks after HIV test results

\begin{tabular}{|c|c|c|c|c|c|c|}
\hline \multirow[b]{2}{*}{ Item 9 on BDI } & \multicolumn{3}{|c|}{ Suicidal ideation 72 hours after HIV diagnosis* } & \multicolumn{3}{|c|}{ Suicidal ideation 6 weeks after HIV diagnosis ${ }^{\dagger}$} \\
\hline & No & Yes & Total & No & Yes & Total \\
\hline 0 & $108(82.4 \%)$ & $23(17.6 \%)$ & 131 & $75(75 \%)$ & $25(25.0 \%)$ & 100 \\
\hline 1 & $16(64 \%)$ & $9(36 \%)$ & 25 & $3(33.3 \%)$ & $6(66.7 \%)$ & 9 \\
\hline Total & $124(79.5 \%)$ & $32(20.5 \%)$ & 156 & $78(71.6 \%)$ & $31(28.4 \%)$ & 109 \\
\hline $\begin{array}{l}{ }^{*} p=0.036 . \\
{ }^{p} p=0.0008 .\end{array}$ & & & & & & \\
\hline
\end{tabular}




\begin{tabular}{|c|c|c|c|c|c|c|}
\hline \multirow[b]{2}{*}{ Item 7 on BHS } & \multicolumn{3}{|c|}{ Suicidal ideation 72 hours after HIV diagnosis* } & \multicolumn{3}{|c|}{ Suicidal ideation 6 weeks after HIV diagnosis ${ }^{\dagger}$} \\
\hline & No & Yes & Total & No & Yes & Total \\
\hline 0 & $52(92.9 \%)$ & $4(7.1 \%)$ & 56 & $55(100 \%)$ & $0(0 \%)$ & 55 \\
\hline 1 & $72(72.0 \%)$ & $28(28.0 \%)$ & 100 & $24(42.9 \%)$ & $32(57.1 \%)$ & 56 \\
\hline Total & $124(79.5 \%)$ & 32 (20.5\%) & 156 & 79 (71.2\%) & 32 28.8\%) & 111 \\
\hline
\end{tabular}

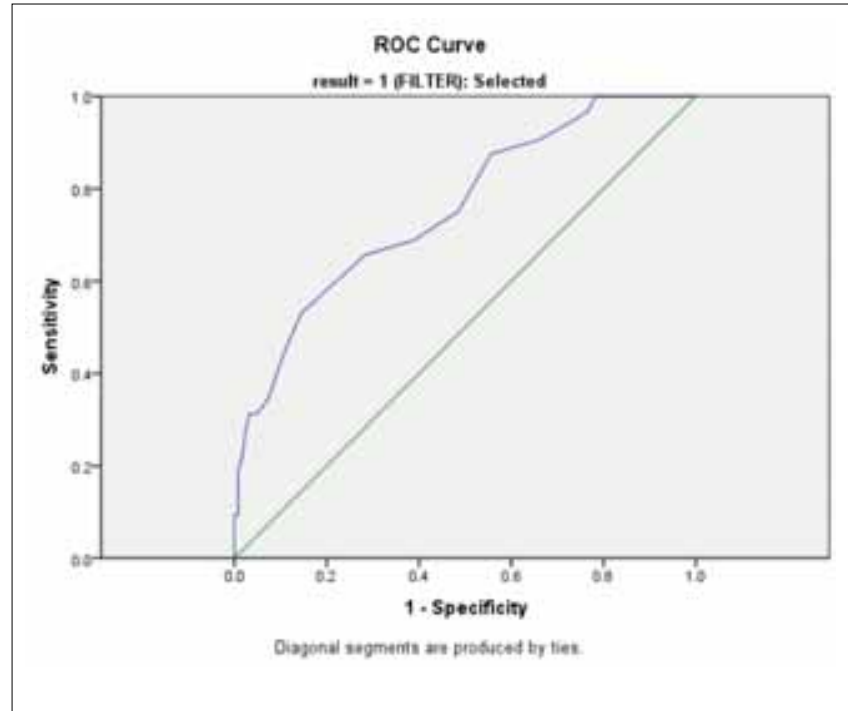

Fig 1. ROC curve of BDI scores v. suicidal ideation (BHS scores) in HIV-positive patients 72 hours after HIV diagnosis.

Fig. 1 indicates the ROC curve of the BDI scores used to predict suicidal ideation at 72 hours after HIV diagnosis versus suicidal ideation on the BHS scores. The AUC was $0.757(p<0.001)$, and the $\mathrm{BDI}$ score was therefore considered a good predictor of suicidal ideation at 72 hours.

Table 3 shows the cut-off points of the BDI and their corresponding sensitivity, specificity and percentage correctly classified, as well as the positive and negative likelihood ratios (LR) at 72 hours after HIV diagnosis. Using this table, it can be seen that a cut-off score of 10 ( $\geq 10$ being a positive result) achieves 100\% sensitivity and $21.8 \%$ specificity in predicting suicidal ideation.

Fig. 2 indicates the ROC curve of BDI scores used to predict suicidal ideation at 6 weeks after HIV diagnosis versus suicidal ideation on the BHS scores. The AUC was $0.788(p<0.001)$, so the BDI scores were considered a good predictor of suicidal ideation at 6 weeks.

Table 4 shows the cut-off points of the BDI scores and their corresponding sensitivity, specificity and percentage correctly classified, as well as the positive and negative likelihood ratios at 6

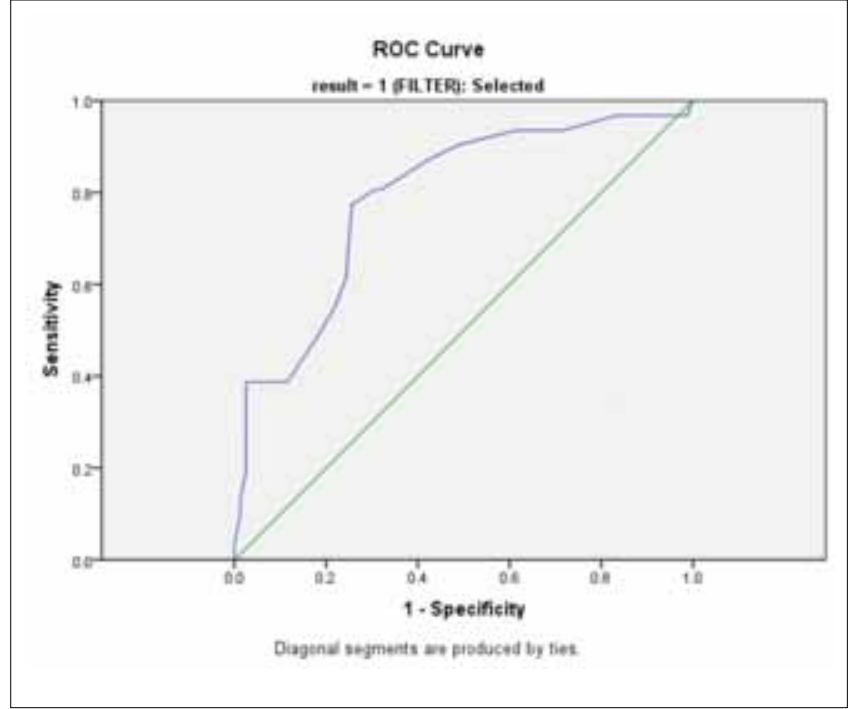

Fig 2. ROC curve of BDI scores v. suicidal ideation (BHS scores) in HIV-positive patients 6 weeks after HIV diagnosis.

weeks. Here the sensitivity drops off at very low values of the BDI scores compared with those at 72 hours. Using this table, it can be seen that a cut-off score of 9 ( $\geq 9$ being a positive result) achieves $96.77 \%$ sensitivity and $16.67 \%$ specificity in predicting suicidal ideation. A cut-off point of 10 on the BDI at 72 hours and 9 at 6 weeks after HIV diagnosis therefore showed optimal sensitivity in indicating that the depressed patients in this study were at risk for suicide, although the specificities were low. These cut-off points serve to flag suicide ideators (and possible suicide attempters) who would benefit from intervention measures aimed at suicide prevention. ${ }^{26}$ According to these results, the BDI can be used to predict suicidal ideation in the sample studied.

\section{Discussion}

The findings in this study confirm those of earlier studies ${ }^{4-5,27-29}$ that many HIV-positive persons have high levels of depressive symptomatology, as well as the fact that those with suicidal ideation reported increased levels of such symptoms. Beck's cognitive theory of depression ${ }^{6,30}$ can be used to contextualise this relationship in that the cognitive symptoms of depression tend to precede the affective and mood components of the disorder, which in 


\begin{tabular}{|c|c|c|c|c|c|}
\hline Cut point & Sensitivity (\%) & Specificity (\%) & Correctly classified (\%) & Likelihood ratio + & Likelihood ratio - \\
\hline$(\geq 0)$ & 100.00 & 0.00 & 20.51 & 1.0000 & \\
\hline$(\geq 3)$ & 100.00 & 1.61 & 21.79 & 1.0164 & 0.0000 \\
\hline$(\geq 4)$ & 100.00 & 2.42 & 22.44 & 1.0248 & 0.0000 \\
\hline$(\geq 5)$ & 100.00 & 5.65 & 25.00 & 1.0598 & 0.0000 \\
\hline$(\geq 6)$ & 100.00 & 6.45 & 25.64 & 1.0690 & 0.0000 \\
\hline$(\geq 7)$ & 100.00 & 8.87 & 27.56 & 1.0973 & 0.0000 \\
\hline$(\geq 8)$ & 100.00 & 11.29 & 29.49 & 1.1273 & 0.0000 \\
\hline$(\geq 9)$ & 100.00 & 16.13 & 33.33 & 1.1923 & 0.0000 \\
\hline$(\geq 10)$ & 100.00 & 21.77 & 37.82 & 1.2784 & 0.0000 \\
\hline$(\geq 11)$ & 96.88 & 23.39 & 38.46 & 1.2645 & 0.1336 \\
\hline$(\geq 12)$ & 93.75 & 28.23 & 41.67 & 1.3062 & 0.2214 \\
\hline$(\geq 13)$ & 90.63 & 33.87 & 45.51 & 1.3704 & 0.2768 \\
\hline$(\geq 14)$ & 87.50 & 44.35 & 53.21 & 1.5725 & 0.2818 \\
\hline$(\geq 15)$ & 75.00 & 51.61 & 56.41 & 1.5500 & 0.4844 \\
\hline$(\geq 16)$ & 71.88 & 56.45 & 59.62 & 1.6505 & 0.4982 \\
\hline$(\geq 17)$ & 68.75 & 61.29 & 62.82 & 1.7760 & 0.5099 \\
\hline$(\geq 18)$ & 65.63 & 71.77 & 70.51 & 2.3250 & 0.4789 \\
\hline$(\geq 19)$ & 53.13 & 85.48 & 78.85 & 3.6597 & 0.5483 \\
\hline$(\geq 20)$ & 43.75 & 89.52 & 80.13 & 4.1731 & 0.6284 \\
\hline$(\geq 21)$ & 34.38 & 92.74 & 80.77 & 4.7361 & 0.7076 \\
\hline$(\geq 22)$ & 31.25 & 95.16 & 82.05 & 6.4583 & 0.7225 \\
\hline$(\geq 23)$ & 31.25 & 96.77 & 83.33 & 9.6875 & 0.7104 \\
\hline$(\geq 24)$ & 28.13 & 97.58 & 83.33 & 11.6250 & 0.7366 \\
\hline$(\geq 25)$ & 21.88 & 98.39 & 82.69 & 13.5625 & 0.7941 \\
\hline$(\geq 27)$ & 18.75 & 99.19 & 82.69 & 23.2500 & 0.8191 \\
\hline$(\geq 28)$ & 15.63 & 99.19 & 82.05 & 19.3750 & 0.8506 \\
\hline$(\geq 30)$ & 9.38 & 99.19 & 80.77 & 11.6250 & 0.9136 \\
\hline$(\geq 31)$ & 9.38 & 100.00 & 81.41 & & 0.9063 \\
\hline$(\geq 32)$ & 6.25 & 100.00 & 80.77 & & 0.9375 \\
\hline$(\geq 34)$ & 3.13 & 100.00 & 80.13 & & 0.9688 \\
\hline$(>34)$ & 0.00 & 100.00 & 79.49 & & 1.0000 \\
\hline
\end{tabular}

turn are triggered by negative automatic thoughts in suicidal behaviour. ${ }^{31-35}$ The sequelae can range from a major depressive disorder precipitated by progression of the illness to a depressive episode secondary to the biopsychosocial issues related to being infected with the HI virus. A positive HIV diagnosis may be viewed by some as a negative life event resulting in, among others, marital problems, financial problems, stigmatisation by family, friends and community, fear of disclosure of a positive HIV test result, problems in accessing health care, and other difficulties., 5,14,34 Consequently, feelings of hopelessness, symptoms of depression and in some patients, suicidal ideation arise. Hopelessness is a primary mediator that links depression and suicidal ideation, and the more hopeless the individual feels about the future, the more depressed they are likely to become, ${ }^{30}$ i.e. it results in the 'revolving door' effect unless appropriate interventions are implemented. The majority of the HIV-positive patients in this study felt that the future seemed dark to them, irrespective of whether or not they had suicidal ideation.

It was estimated that only about 193579 South Africans were receiving antiretroviral therapy by the end of 2005, ${ }^{28}$ and many affected South Africans may believe that they have little hope of obtaining ongoing antiretroviral treatment. A worrying observation in the present study is the correlation between item 7 on the BHS ('My future looks dark') and suicidal ideation 6 weeks after a positive 
HIV diagnosis. Initially (i.e. pre-VCT) the future may have looked different, but with positive test results, reality sets in and the patients may have begun to envisage their future with less hope. A model of the link between a positive HIV diagnosis, hopelessness, depression and the potential risk for suicidal ideation is reflected in the flow diagram in Fig. 3.

Fortunately hopelessness and depression are both measurable and modifiable psychological variables, and unlike some other precursors of suicidal behaviour can therefore be timeously assessed. An advantage of using psychometric measures in this regard is that they are reliable yardsticks that, under clinical supervision, can be used by less highly trained health professionals for screening purposes at VCT clinics. The results can be used in treatment strategies to help prevent suicidal behaviour in these patients.

\section{Limitations}

Limitations of this study include the fact that predetermined values for suicidal ideation, as depicted by the measuring instruments utilised, were used. For a more comprehensive suicide risk assessment, additional variables should be included such as, for

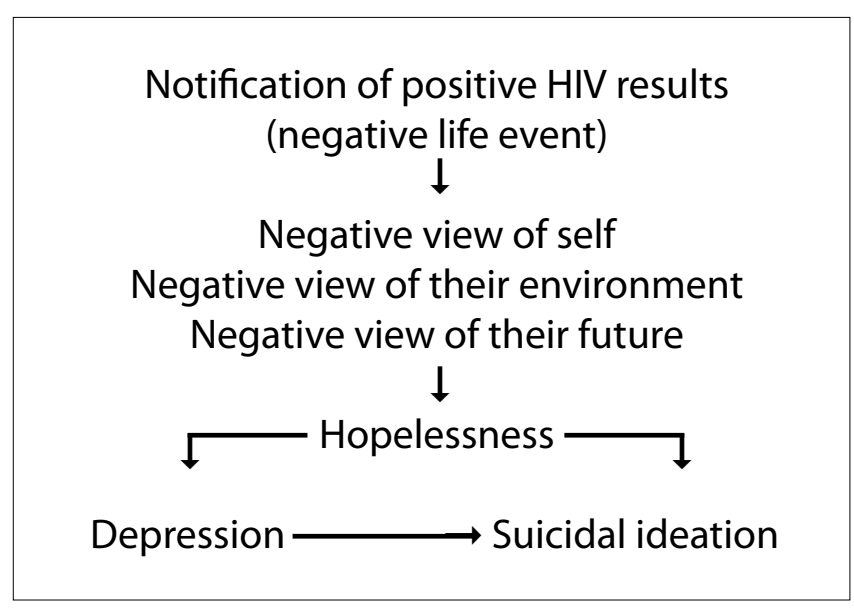

Fig. 3. Flow diagram linking seropositivity, hopelessness, depression and suicidal ideation.

\begin{tabular}{|c|c|c|c|c|c|}
\hline Cut point & Sensitivity & Specificity & Correctly classified & Likelihood ratio + & Likehood ratio - \\
\hline$(\geq 1)$ & 100.00 & 0.00 & 28.44 & 1.0000 & \\
\hline$(\geq 4)$ & 96.77 & 1.28 & 28.44 & 0.9803 & 2.5161 \\
\hline$(\geq 5)$ & 96.77 & 2.56 & 29.36 & 0.9932 & 1.2581 \\
\hline$(\geq 6)$ & 96.77 & 5.13 & 31.19 & 1.0201 & 0.6290 \\
\hline$(\geq 7)$ & 96.77 & 10.26 & 34.86 & 1.0783 & 0.3145 \\
\hline$(\geq 8)$ & 96.77 & 11.54 & 35.78 & 1.0940 & 0.2796 \\
\hline$(\geq 9)$ & 96.77 & 16.67 & 39.45 & 1.1613 & 0.1935 \\
\hline$(\geq 10)$ & 93.55 & 28.21 & 46.79 & 1.3030 & 0.2287 \\
\hline$(\geq 11)$ & 93.55 & 38.46 & 54.13 & 1.5202 & 0.1677 \\
\hline$(\geq 12)$ & 90.32 & 51.28 & 62.39 & 1.8540 & 0.1887 \\
\hline$(\geq 13)$ & 87.10 & 57.69 & 66.06 & 2.0587 & 0.2237 \\
\hline$(\geq 14)$ & 80.65 & 67.95 & 71.56 & 2.5161 & 0.2848 \\
\hline$(\geq 15)$ & 80.65 & 69.23 & 72.48 & 2.6210 & 0.2796 \\
\hline$(\geq 16)$ & 77.42 & 74.36 & 75.23 & 3.0194 & 0.3037 \\
\hline$(\geq 17)$ & 61.29 & 75.64 & 71.56 & 2.5161 & 0.5118 \\
\hline$(\geq 18)$ & 54.84 & 78.21 & 71.56 & 2.5161 & 0.5775 \\
\hline$(\geq 19)$ & 48.39 & 82.05 & 72.48 & 2.6959 & 0.6290 \\
\hline$(\geq 20)$ & 38.71 & 88.46 & 74.31 & 3.3548 & 0.6928 \\
\hline$(\geq 21)$ & 38.71 & 94.87 & 78.90 & 7.5484 & 0.6460 \\
\hline$(\geq 22)$ & 38.71 & 97.44 & 80.73 & 15.0968 & 0.6290 \\
\hline$(\geq 23)$ & 22.58 & 97.44 & 76.15 & 8.8065 & 0.7946 \\
\hline$(\geq 24)$ & 19.35 & 97.44 & 75.23 & 7.5484 & 0.8277 \\
\hline$(\geq 25)$ & 12.90 & 98.72 & 74.31 & 10.0645 & 0.8823 \\
\hline$(\geq 26)$ & 9.68 & 98.72 & 73.39 & 7.5484 & 0.9150 \\
\hline$(\geq 31)$ & 3.23 & 100.00 & 72.48 & & 0.9677 \\
\hline$(>31)$ & 0.00 & 100.00 & 71.56 & & 1.0000 \\
\hline
\end{tabular}


example, a history of previous suicidal behaviour, a family history of suicidal behaviour, a pre-existing history of mood/or other psychiatric disorders, alcohol or drug abuse, and related sociodemographic factors. Because of the nature of the study design, the relationship between seropositivity, hopelessness, depression and an ultimate suicide attempt could not be assessed.

\section{Conclusion}

Suicidal behaviour is a major public health concern in South Africa and elsewhere. ${ }^{5,36-37}$ The presence of hopelessness, depression and suicidal ideation are important psychological variables that should alert healthcare professionals to underlying suicide risks in HIVpositive patients. Early recognition of this and suicide prevention strategies should be incorporated into treatment at VCT HIV clinics.

Acknowledgements. The authors wish to thank the field workers for their excellent work ethic and collection of data for this research. Our sincere gratitude to Mrs T Esterhuizen for the statistical input and to Ms P Pillay for research assistance.

Sponsorship. Dr R D Govender was supported by the Columbia University-South African Fogarty AIDS International Training and Research Programme (AITRP) funded by the Fogarty International Center, National Institutes of Health (grant D43TW00231).

\section{Conflict of interest. None.}

\section{References}

1. Beck AT, Steer RA, Kovacs M, Garrison B. Hopelessness and eventual suicide: a 10-year prospective study of patients hospitalized with suicidal ideation. Am J Psychiatry 1985;142:559-563. [doi: 10.1016/0165-0327(89)90001-3]

2. Cooper-Patrick L, Crum RM, Ford DE. Identifying suicidal ideation in general medical patients. JAMA 1994;272(22):1757-1762. [doi: 10.1001/jama.1994.03520220051030]

3. Perry SW. HIV-related depression. Res Publ Assoc Res Nerv Ment Dis 1994;72:223-228.

4. Meel BL, Leenaars AA. Human immunodeficiency virus (HIV) and suicide in a region of Eastern Province ('Transkei'), South Africa. Arch Suicide Res 2005;9(1):69-75.

5. Schlebusch L. Suicidal Behaviour in South Africa. Pietermaritzburg: University of KwaZuluNatal Press, 2005

6. McIntosh CN, Fischer DG. Beck's cognitive triad: one versus three factors. Canadian Journal of Behavioural Science2000;32:153-157. [doi: 10.1002/j.1556-6676.1995.tb01777.x]

7. Inskip HM, Harris EC, Barraclough B. Lifetime risk of suicide for affective disorder, alcoholism and schizophrenia. Br J Psychiatry 1998;172:35-37. [doi: 10.1192/bjp.172.1.35]

8. Masango SM, Rataemane ST, Motojesi AA. Suicide and suicide risk factors: A literature review. South African Family Practice 2008;50(6):25-29.

9. Wolfersdorf M. Depression and suicide. Bundesgesundheitsblatt Gesundheitsforshung Gesundheitsschutz 2008;51(4):443-450.

10. Kaharuza FM, Bunnel R, Moss S, et al. Depression and CD4 count in persons with HIV infection in Uganda. AIDS Behav 2006;10(suppl):S105-S111.
11. Antelman G, Kaaya $S$, Wei $R$, et al. Depressive symptoms increase risk of HIV disease progression and mortality among women in Tanzania. J Acquir Immune Defic Syndr 2007;44(4):470-477.

12. Antakly de Mello V, Malbergier A. Depression in women infected with HIV. Rev Bras Psiquiatr 2006;28(1):10-17. [doi: 10.1590/S1516-44462006000100004]

13. Schlebusch L, Vawda N. HIV-infection as a self-reported risk factor for attempted suicide in South Africa. African Journal of Psychiatry 2010;13(4):280-283.

14. Kallichman SC, Heckman T, Kochman A, et al. Depression and thoughts of suicide among middle-aged and older persons living with HIV-AIDS. Psychiatr Serv 2000;51 (7):903-907.

15. Beck AT, Brown G, Berchick RJ, Stewart BL, Steer RA. Relationship between hopelessness and ultimate suicide: a replication with psychiatric outpatients. Am J Psychiatry 1990;147(2):190-195.

16. Beck AT, Steer RA, Beck JS, Newman CF. Hopelessness, depression, suicidal ideation, and clinical diagnosis of depression. Suicide and Life-Threatening Behavior 1993;23(2):139-145.

17. Shneidman ES. The Suicidal Mind. Hong Kong: Oxford University Press, 1998.

18. De Jezus Castro SM, Trentini C, Riboldi J. Item response applied to the Beck Depression. Rev Bras Epidemiol 2010;13(3):487-501. [doi: 10.1590/S1415-790X2010000300012]

19. Beck AT, Steer RA, Carbin M. Psychometric properties of Beck Depression Inventory: Twentyfive years of evaluation. Clin Psychol Rev 1988;8(1):77-100

20. Steer RA, Kumar G, Beck AT. Self-reported suicidal ideation in adolescent psychiatric inpatients. J Consult Clin Psychol 1993;61(6):1096-1099.

21. Yip PSF, Cheung YB. Quick assessment of hopelessness: a cross-sectional study. Health Qual Life Outcomes 2006;4(13). [doi:10.1186/1477-7525-4-13]

22. Aish A, Wasserman D. Does Beck's Hopelessness Scale really measure several components? Psychol Med 2001;31:367-372.

23. Grunkemeier GL, Jin R. Receiver operating characteristic curve analysis of clinical risk models. Ann Thorac Surg 2001;72:323-326.

24. Swets JA. ROC analysis applied to the evaluation of medical imaging techniques. Invest Radiol 1979;14:365-377.

25. Fleischmann A, Bertolote José M, Wasserman D, et al. Effectiveness of brief intervention and contact for suicide attempters: a randomized controlled trial in five countries. Bull World Health Organ 2008;86(9):703-709. [doi: 10.1590/50042-96862008000900014]

26. Keogh $\mathrm{P}$, Alle S, Almedal C, et al. The social impact of HIV on women in Kigali, Rwanda: a prospective study. Soc Sci Med 1994;38:1047-1053.

27. Nattrass N. South Africa's 'rollout' of highly active antiretroviral therapy: A critical assessment. J Acquir Immune Defic Syndr 2006;43(5):618-623.

28. Judd FK, Mijch AM. Depressive symptoms in patients with HIV infection. Aust N Z Psychiatry 1996;30(1):104-109.

29. Beck AT. Depression: Clinical, Experimental and Theoretical Aspects. New York: Harper \& Row, 1967

30. Beck AT, Kovacs M, Weissman A. Hopelessness and suicidal behavior. An overview. JAMA 1975; 34(11):1146-1149

31. Schlebusch L, Wessels WH. Hopelessness and low-intent in parasuicide. Gen Hosp Psychiatry 1988;10(3):209-213.

32. Beck AT, Weissman A, Lester D, Trexler L. The measurement of pessimism: the Hopelessness Scale. J Consult Clin Psychol 1974;42:861-865.

33. Vance DE, Struzick T, Childs G. Challenges of depression and suicidal ideation associated with aging with HIV/AIDS: implications for social work. J Gerontol Soc Work 2010;53(2):159175.

34. Beck AT, Steer RA, Beck JS, Newman CF. Hopelessness, depression, suicidal ideation, and clinical diagnosis of depression. Suicide Life Threat Behav 1993;23(2):139-145.

35. Schlebusch L. Current perspectives on suicidal behaviour in South Africa. In: Suffle S, Van Niekerk A, Duncan D, eds. Crime, Violence and Injury Prevention in South Africa: Developments and Challenges. Tygerberg, W Cape: Medical Research Council-UNISA, 2004:88-113.

36. Schlebusch L. Depression and suicidal behaviour. South African Family Practice 2005;47(5):61-63.

37. Wasserman D. Wasserman C, eds. The Oxford Textbook of Suicidology and Suicide Prevention. A Global Perspective. Oxford: Oxford University Press, 2009 